\title{
Simulation and Experimental Investigation of Pulsed Eddy Current Technique for Defect Evaluation
}

\author{
Nurul A'in Ahmad Latif ${ }^{1}$, Ilham Mukriz Zainal Abidin ${ }^{2}$, Nordin Jamaludin ${ }^{3}$ \\ ${ }^{1,3}$ Department of Mechanical and Materials Engineering, Faculty of Engineering and Built Environment, \\ Universiti Kebangsaan Malaysia, 43600 Bangi, Selangor, Malaysia \\ ${ }^{1,2}$ Leading Edge NDT Technology (LENDT) Group, Industrial Technology Division, Malaysian Nuclear Agency, \\ 43000 Bangi Selangor, Malaysia
}

\begin{tabular}{l}
\hline \hline Article Info \\
\hline Article history: \\
Received May 22, 2018 \\
Revised Jul 31, 2018 \\
Accepted Sep 6, 2018
\end{tabular}

Keyword:

Crack

FEM simulation

Magnetic field

Non-destructive testing

Pulsed eddy current

\begin{abstract}
Pulsed eddy current (PEC) is an advanced Non-Destructive Testing (NDT) technique that uses transient waveform for their coil excitation. It has the advantage of gathering different depth information in a single excitation process, thus provide the solution towards the drawbacks by both single and multi-frequency eddy current testing. In this work, experimental and simulation investigations on PEC were conducted to establish the correlation between PEC signals and different defect i.e. crack, depths. Initial FEM simulation work has provided understanding of the underlying phenomena of the PEC results through the visualisation of the induced eddy current and defect interaction in the SS 304 sample. Features from the differential magnetic field transient profiles have provided information of the crack in terms of its depth and location. The understanding of the PEC responses and results can be extended for future work in quantitative evaluation of defect in conductive samples by PEC.
\end{abstract}

Copyright $@ 2018$ Institute of Advanced Engineering and Science. All rights reserved.

Corresponding Author:

Nurul A'in Ahmad Latif,

Department of Mechanical and Materials Engineering,

Faculty of Engineering and Built Environment,

Universiti Kebangsaan Malaysia,

43600 Bangi, Selangor, Malaysia

Email: imaancouture@gmail.com

\section{INTRODUCTION}

Eddy current testing is one of the Non-Destructive Testing (NDT) methods which is based on electromagnetic principles and has been effectively been used on electrically conductive samples. It allows the inspection of surface breaking as well as subsurface defects, due to the interaction between the induced eddy currents with the defects [1]-[3]. In eddy current testing, the excitation frequency is selected based on the sample and the depths of the defects to be detected. In general, the coil use in eddy current testing is operated by a single frequency sinusoidal excitation and the signal is presented on an impedance plane diagram [4].

To enhance the inspection capability in eddy current testing, the multi-frequency excitation has been introduced [5],[6]. However, the limitation of eddy current penetration in both single and multi-frequency eddy current testing has become the main drawback in its application [7]. To counteract this limitation, the pulsed eddy current (PEC) technique has been introduced. This technique is based on pulsed (square or rectangular) excitation current and provides new perspectives for the detection and characterisation of defects in test samples through the measurement of transient response of the magnetic field [8].

The wideband pulse in PEC excitation consists of a series of frequency components leading to richness of information gathered about the defect [9]-[11]. The technique has the advantage that it is equivalent to a multitude of single frequency with simultaneous injection of sinusoidal inputs [12],[13]. In 
PEC, the signals are represented in the time domain, rather than the more familiar impedance plane diagram, as is the case for conventional eddy current testing. One of the advantages of PEC over conventional ET is the replacement of pick up coils with magnetic field sensor. Magnetic sensor offers the high broadband sensitivity over a wide band of frequencies. In this paper, Hall sensor was used due to its ability to provide a high sensitivity to distinguish a small differences of defects' depth. Although PEC is a popular research technique, so far it has not been widely accepted in industry, mainly due to the difficulty to interpret and quantify the inspection signals [14].

In order to get better understanding of the PEC results especially for the quantitative evaluation of defects, simulation and experimental works are conducted to establish the correlation between PEC signals and different defect i.e. crack, depths. Such investigations would be very helpful in understanding and interpreting the PEC responses though the transient profiles of the magnetic flux density from the eddy current and crack interaction in the sample. This will facilitates not only forward problem but also inverse solution [15] for 3D reconstruction [16] and pattern recognition [17] of defects through the establishment of linkages between experiment and simulation [18].

The paper is organized as a follows: Section 2.0 presents the model setup and results for PEC simulation; Section 3 presents the PEC system and experimental results; Section 4 provides the conclusion of this work.

\section{VISUALISATION AND CORRELATION OF PEC RESPONSES}

\subsection{Simulation Model and Setup}

A series of finite element method (FEM) numerical modelling simulations are conducted to build the relationship between the acquired magnetic field transient profiles and the induced eddy currents with different crack geometrical characteristic i.e. depth. Through the simulation, the underlying phenomena of PEC can be understood and the acquired results can be explained through the visualisation of the induced eddy currents inside the tested sample. To implement this study, a PEC coil was modelled with dimensions of $18 \mathrm{~mm}$ outer diameter, $12.5 \mathrm{~mm}$ inner diameter, $35 \mathrm{~mm}$ height with 350 turns of wire, and is current driven with a rectangular waveform of $1 \mathrm{~A}$ amplitude to generate a varying magnetic field.

A $20 \mathrm{~mm}$ thick stainless steel sample with different crack depths of $2 \mathrm{~mm}, 4 \mathrm{~mm}, 6 \mathrm{~mm}, 8 \mathrm{~mm}$ and $10 \mathrm{~mm}$ were introduced in the study. A relative thick sample is used to enable good visualisation of the simulated induced eddy currents and thus observe the difference in the net transient magnetic field profiles.In the time-stepping FEM, the transient magnetic field profiles were acquired when the coil is positioned above the crack opening. The time step of the pulse waveform is $10 \mathrm{~ms}$ with $5 \mathrm{~ms}$ excitation pulse width and pulse repetition frequency is $100 \mathrm{~Hz}$. All simulations were performed using COMSOL. Figure 1 shows the layout of the coil and the stainless steel sample having a defect, modelled as a 3D problem.

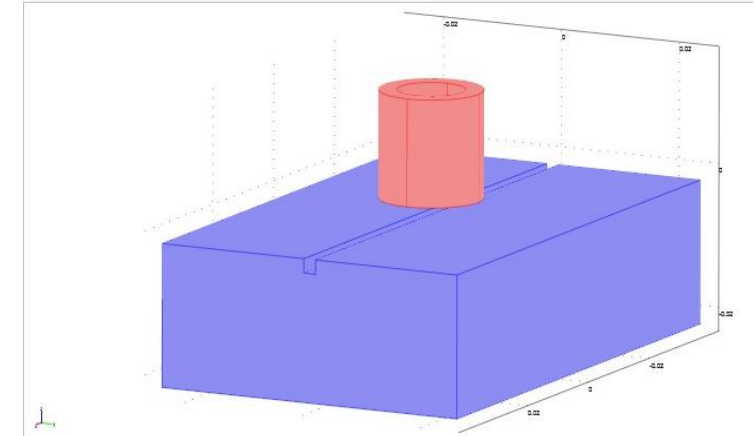

Figure 1.3D model of the PEC coil and stainless steel with a defect

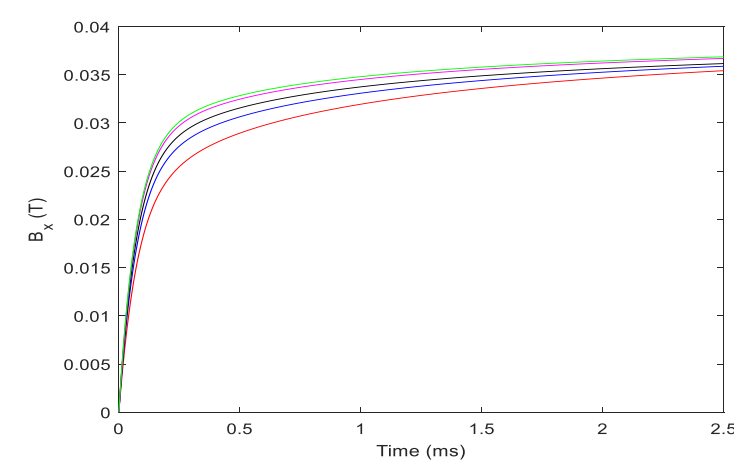

Figure 2. Net magnetic field, $B_{x}$ acquired at different crack depths

For a cylindrical PEC coil located over a conductive sample, the magnetic field, within the vicinity of the sample is given by equation 1 .

$$
\mathrm{B}_{\mathrm{x}}=\mathrm{B}_{\mathrm{z}}^{\mathrm{e}}-\Delta \mathrm{B}_{\mathrm{z}}
$$


where $B_{x}$ denotes the magnetic field at x-component, $B_{z}^{e}$ is the field produced by the coil and $\Delta B_{z}$ is the field change caused by the sample.

\subsection{PEC Transient Profiles}

Figure 2 shows the acquired transient profiles for different crack depths. These are the resultant net magnetic field from the interaction between the primary magnetic field coming from the coil and the secondary magnetic field produced by the induced eddy current inside the stainless steel sample. Crack with $10 \mathrm{~mm}$ depth exhibits the highest followed by the $8 \mathrm{~mm}, 6 \mathrm{~mm}, 4 \mathrm{~mm}$ and $2 \mathrm{~mm}$, in the respective order. The results show that as the depth of the crack increases, the amplitude of the respective profiles increases.

As we know, the acquired $B_{x}$ is in close relation with the induced eddy current density as well as its distribution. Therefore, in order to fully understand the underlying phenomena of the PEC results, the visualisation of the induced eddy current with different crack depths were acquired from the simulation. The cross section view of the stainless steel sample for every crack depths are presented in Figure 3 . This signals were acquired at steady state of transient signal at $2.5 \mathrm{~ms}$. From the visualisation provided by the simulation, it can be observed that the eddy current density is changing with different crack depths. The presence of a crack inside the stainless steel sample will perturb the flow of the induced eddy currents and changes its overall density at that particular area. With the increase of crack depths, the flow of the eddy currents will increasingly be perturbed thus results in reduced in eddy current density as shown in Figure 3a to Figure 3e. This in turn increase the net $B_{x}$ acquired from the sample by the PEC coil with increase crack depth, as presented in Figure 2.

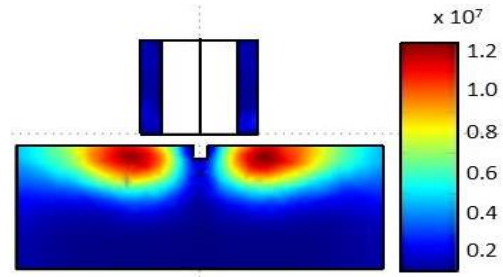

(a)

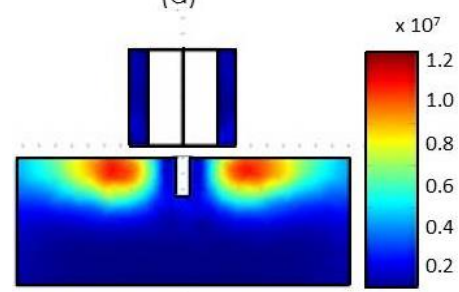

(c)

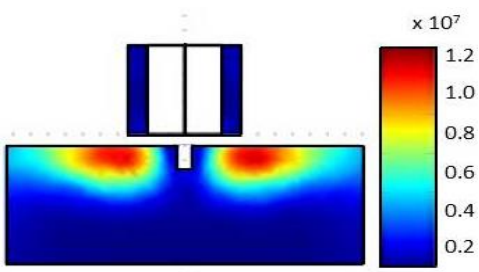

(b)

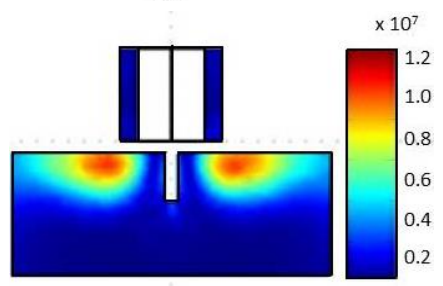

(d)

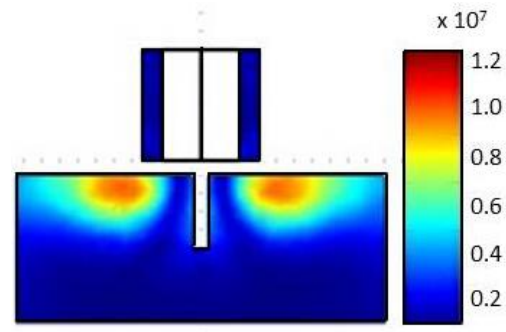

(e)

Figure 3. Simulated visualisation of eddy current density at different crack depth of a) $2 \mathrm{~mm}$, b) $4 \mathrm{~mm}, \mathrm{c}) 6$ $\mathrm{mm}$, d) 8 and e) $10 \mathrm{~mm}$

\section{METHODOLOGY}

\subsection{PEC Crack Depth Evaluation}

A PEC system consists of a function generator, power amplifier, power supply and a PEC coil is used to conduct the PEC investigation towards different crack depths. Figure 4 presents the illustration and the developed PEC system for the work. The power supply supplies the voltage to power the magnetic sensor. The function generator provides the rectangular input current to drive the excitation coil. In this work,

\footnotetext{
Simulation and Experimental Investigation of Pulsed Eddy Current Technique .... (Nurul A'in Ahmad Latif)
} 
a waveform with a $5 \mathrm{~ms}$ pulse width is used for the excitation. For the signals processing, all the signals acquired by the sensor will be transferred, displayed and processed using the computer which equipped with a data acquisition card.

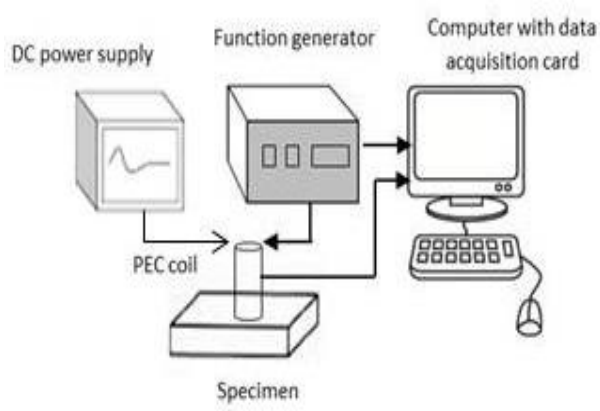

(a)

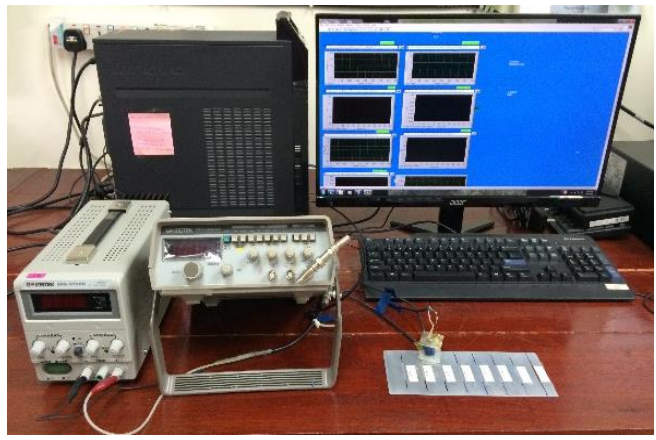

(b)

Figure 4. (a) Schematic diagram of a PEC system; (b) The PEC system developed for the work

Figure 5 provide the details of the PEC coil associated with the system. The PEC coil has the dimensions of $16 \mathrm{~mm}$ outer diameter, $10.5 \mathrm{~mm}$ inner diameter, $30 \mathrm{~mm}$ height and 300 turns of copper wire which is wound on a ferrite core (Figure 5a). A magnetic sensor (Hall SS490) is utilised and placed at the centre of the ferrite core, which function as a sensor to acquire the magnetic field.

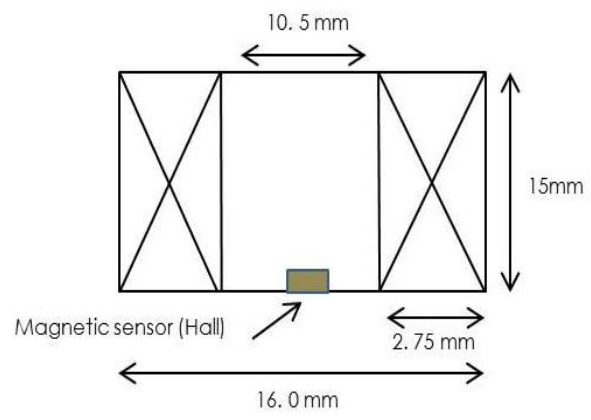

Figure 5. Schematic diagram of PEC coil

The sample under investigation is a $5 \mathrm{~mm}$ in thickness stainless steel 304 plate containing open to surface cracks (EDM machined) with depths, $d=0.15 \mathrm{~mm}, 0.20 \mathrm{~mm}, 0.25 \mathrm{~mm}, 0.30 \mathrm{~mm}, 0.35 \mathrm{~mm}, 0.40 \mathrm{~mm}$, $0.50 \mathrm{~mm}$ and having an opening of $2 \mathrm{~mm}$. This relative thin sample provides a challenge in differentiating the difference in depths for the defect. For the experiment and simulation works the signals were acquired when the probe is positioned above the crack opening with a lift-off of $2 \mathrm{~mm}$. Figure 6 shows the stainless steel sample with cracks having different depths used in the experiment.

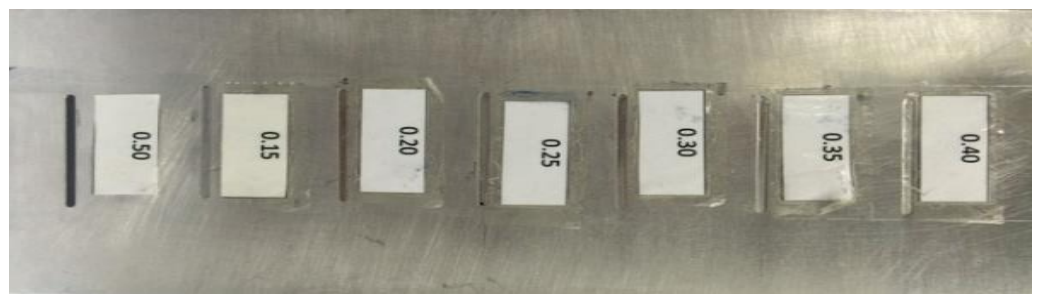

Figure 6. Stainless steel plate containing EDM machined cracks with different depths 
The magnitude of change in the acquired net $B_{x}$ is relatively small, especially with cracks within thin samples. To observe the change of the field with the change of the crack depths, the differential net magnetic field profile, $\Delta B_{x}$ were obtained by taking the difference between the transient net $B_{x}$ from a reference signal and of that of the stainless steel sample at each crack depth. The $\Delta B_{x}$ for all crack depths can be mathematically defined as follows:

$$
\Delta \mathrm{B}_{\mathrm{x}}=\mathrm{B}_{\mathrm{xref}}-\mathrm{B}_{\mathrm{xm}}
$$

where $B_{x r e f}$ is the reference magnetic field, $B_{x m}$ is the net magnetic field acquired from the stainless steel sample and represents the cracks with depths $=0.15 \mathrm{~mm}, 0.20 \mathrm{~mm}, 0.25 \mathrm{~mm}, 0.30 \mathrm{~mm}, \ldots, \mathrm{N} \mathrm{mm}$.

Figure 7a presents the acquired transient $B_{x}$ profiles by the PEC coil from the experiment. The results show a good agreement with the simulation (section 2.1), whereby as the depth of the crack increases, the amplitude of the respective profiles increases. It can be seen from the figure, $B_{x}$ for air has the highest amplitude compared to other acquired PEC signals. Subsequently, $\Delta B_{x}$ for each crack with different depth were attained against air, which act as the reference signal. The $\Delta B_{x}$ profiles for all crack depths are plotted and presented in Figure $7 \mathrm{~b}$.

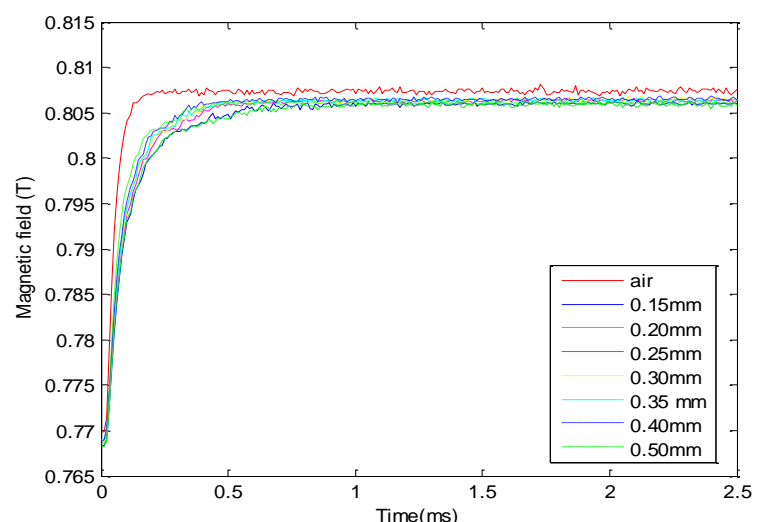

(a)

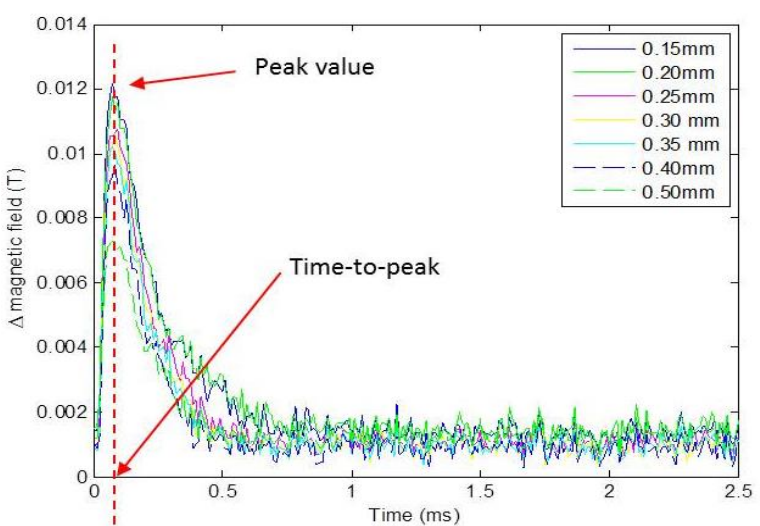

(b)

Figure 7. Stainless steel plate containing cracks with different depths

Features from the plotted $\Delta B_{x}$ profiles can be extracted to provide quantitative information of the defect. Such features are the peak value and the time-to-peak of the $\Delta B_{x}$ profiles as shown in Figure $7 \mathrm{~b}$. The peak value and the time-to-peak can be correlated to the crack depth and the location of the crack within the thickness of the sample [19]-[21]. Consequently, it can be observed that the peak values of the resultant $\Delta B_{x}$ (Figure 7b) are reducing with the increase of crack depths. On the other hand, the time-to-peak for each crack depths occurred at the same specific time i.e. $0.07 \mathrm{~ms}$. Since all cracks introduced in this work are open to surface type of defect, thus the cracks are detected to be at the same particular location.

\section{CONCLUSION}

This paper has presented the work on PEC technique and the correlation between acquired PEC signals with cracks having different depths in a stainless steel plate sample. Through the FEM simulation, the underlying phenomena of the PEC responses and the relationship between the acquired magnetic field transient profiles with the induced eddy currents have been explained. The visualizations of the induced eddy currents from the simulation provide forward solution for PEC investigation and thus the inverse approach for crack quantification through the acquired magnetic field transient profiles. Good agreement between the results from the FEM simulation and experiment conducted through the developed PEC system may benefits future work on PEC for real-time quantitative defect evaluation by an inverse algorithm. 


\section{ACKNOWLEDGEMENT}

The authors would like to express gratitude to the Ministry of Science, Technology and Innovation (MOSTI) and the Malaysian Nuclear Agency for funding this work under the Sciencefund grant (03-03-01SF0220) and the support given for this work.

\section{REFERENCES}

[1] C. V. Dodd and W. E. Deeds, "Analytical Solutions to Eddy Current Probe-Coil Problems," Journal of Applied Physics, vol/issue: 39(6), pp. 2829-2838, 1968.

[2] N. Harfield and J. R. Bowler, "Theory of Thin-Skin Eddy Current Interaction with Surface Cracks," Journal of Applied Physics, vol/issue: 82(9), pp. 4590-4603, 1997.

[3] J. R. Bowler and N. Harfield, "Evaluation of Probe Impedance Due to Thin-Skin Eddy Current Interaction with Surface Cracks," IEEE Transactions on Magnetics, vol/issue: 34(2), pp. 515-523, 1998.

[4] J. Hansen, "The Eddy Current Inspection Method, Part 1," History and Electrical Theory. Insight, vol/issue: 46(5), pp. 279-281, 2004.

[5] R. Grimberg, et al., "2D eddy current sensor array," NDT \& E International, vol/issue: 39(4), pp. 264-271, 2006.

[6] L. H. Shu, et al., "Development of Differential Probes in Pulsed Eddy Current Testing for Noise Suppression," Sensors and Actuators A, vol/issue: 135(2), pp. 675-679, 2007.

[7] F. Thollon, et al., "Numerical and Experimental Study of Eddy Current Probes in NDT of Structures with Deep Flaws," NDT International, vol/issue: 28(2), pp. 97-102, 1995.

[8] I. Mukriz, et al., "3D Transient Magnetic Field Mapping for Angular Slots in Aluminium," Insight, vol/issue: 51(1), pp. 21-24, 2009.

[9] G. Y. Tian, et al., "Pulsed Eddy Current System for Dynamic Inspection of Defects," Insight, vol/issue: 46(5), pp. 256-260, 2004.

[10] G. Y. Tian and A. Sophian, "Defect Classification Using a New Feature for Pulsed Eddy Current Sensors," NDT \& E International, vol/issue: 38(1), pp. 77-82, 2005.

[11] R. A. Smith and G. R. Hugo, "Transient Eddy Current NDE for Ageing Aircraft - Capabilities and Limitations," Insight, vol/issue: 43(1), pp. 14-25, 2001.

[12] A. Sophian, et al., "Design of a Pulsed Eddy Current Sensor for Detection of Defects in Aircraft Lap-joints," Sensors and Actuators A: Physical, vol/issue: 101(1-2), pp. 92-98, 2002.

[13] C. Mandache and J. H. V. Lefebvre, "Transient and Harmonic Eddy Currents: Lift-Off Point of Intersection," NDT \& E International, vol/issue: 39(1), pp. 57-60, 2006.

[14] T. W. Krause, et al., "Diffusion of Pulsed Eddy Currents in Thin Conducting Plates," Review of Progress in Quantitative Nondestructive Evaluation, ed. Thompson DO, Chimenti DE. AIP Conference Proceedings, vol. 975, pp. 368-375, 2008.

[15] I. Z. Abidin, et al., "Defect Depth Estimation using Pulsed Eddy Current with Varied Pulse Width Excitation," Insight, vol/issue: 51(2), pp. 1-4, 2009.

[16] N. Ida and W. Lord, "A Finite Element Model for Three-Dimensional Eddy Current NDT Phenomena," IEEE Transactions on Magnetics, vol/issue: 21(6), pp. 2635-2643, 1985.

[17] M. Tanaka and H. Tsuboi, "Finite Element Model of Natural Crack in Eddy Current Testing Problem," IEEE Transactions on Magnetics, vol/issue: 37(5), pp. 3125-3128, 2001.

[18] Y. Li, et al., "Magnetic Field-Based Eddy Current Modeling for Multilayered Specimens," IEEE Transactions on Magnetics, vol/issue: 43(11), pp. 4010-4015, 2007.

[19] G. Y. Tian, et al., "The Research of Inhomogeneity in Eddy Current Sensors," Sensors and Actuators A: Physical, vol/issue: 69(2), pp. 148-151, 1998.

[20] G. Y. Tian and A. Sophian, "Reduction of lift-off effects for pulsed eddy current NDT," NDT \& E International, vol. 38, pp. 319-324, 2005.

[21] A. Sophian, et al., "A Feature Extraction Technique Based on Principle Component Analysis for Pulsed Eddy Current NDT," NDT \& E International, vol. 36, pp. 37-41, 2003. 